\title{
Una especie nueva de Cheiropteronema (Nematoda, Molineidae) en Artibeus planirostris (Chiroptera, Phyllostomidae) en la Argentina
}

\author{
Mirna C. Oviedo ${ }^{1}$, Geraldine Ramallo² \& Lucía E. Claps ${ }^{1,3}$ \\ 1. Instituto Superior de Entomología "Abraham Willink" y Cátedra de Invertebrados, Facultad de Ciencias Naturales e Instituto Miguel \\ Lillo, Universidad Nacional de Tucumán, Tucumán, Argentina. (mirnaoviedo@gmail.com) \\ 2. Instituto de Invertebrados, Fundación Miguel Lillo, Miguel Lillo 251, 4000 San Miguel de Tucumán, Argentina. \\ 3. Facultad de Ciencias y Tecnología, Universidad Autónoma de Entre Ríos, sede Diamante, Entre Ríos, Argentina.
}

\begin{abstract}
A new species of Cheiropteronema (Nematoda, Molineidae) in Artibeus planirostris (Chiroptera, Phyllostomidae) from Argentina. Cheiropteronema striatum sp. nov. is a parasite of the bat Artibeus planirostris (Spix, 1823). This species is characterized by cephalic vesicle striate, thick egg shell punctate, spicules equal, symmetrical development of spicule wings, particular arrangement and number the ridges of synlophe, and lack of esophageal tooth. Cheiropteronema striatum sp. nov. is the second species described for the genus and the first recorded in frugivorous bats of Argentina.
\end{abstract}

KEYWORDS. Frugivorous bats, nematode parasites, Neotropical.

RESUMEN. Se describe a Cheiropteronema striatum sp. nov. parásita del murciélago Artibeus planirostris (Spix, 1823). Se caracteriza por presentar vesícula cefálica estriada, superficie de los huevos con puntuaciones, espículas iguales, nacimiento simétrico del ala espicular, particular disposición y número de crestas del synlophe y por carecer de diente esofágico. Es la segunda especie del género y la primera que se registra en quirópteros frugívoros de la Argentina.

PALABRAS CLAVE. Murciélagos frugívoros, nematodos parásitos, Neotropical.

En Argentina se registran tres especies del quiróptero Artibeus Leach, 1821: A. fimbriatus Gray 1838, A. lituratus (Olfers, 1818) y A. planirostris (Spix, 1823) (BARQUEZ et al., 1999; BARQUEZ et al., 2006).

De ellas, A. planirostris se distribuye en América del Sur, desde el sureste de Colombia, Venezuela y Guyanas hasta el noroeste argentino (BARQUEZ et al., 1999; Hollis, 2005). Esta especie de murciélago es la única del género registrada en el noroeste argentino. Es frecuente pero poco abundante, habita naturalmente los bosques hasta el sur de la provincia de Tucumán, aunque también ha sido capturada en la región Chaqueña (BARQuez \& OJeDA, 1992; BARQUEZ et al., 1999; BARQUEZ \& Díaz, 2001). Asimismo, este quiróptero dispersa las semillas de numerosas plantas colonizadoras y de algunas exóticas, por lo que cumple un papel importante en el mantenimiento de la heterogeneidad de las selvas (Hollis, 2005).

Por otro lado, Cheiropteronema Sandground, 1929, es un género del Nuevo Mundo con una única especie, C. globocephala Sandground, 1929 que parasita a murciélagos frugívoros de la familia Phyllostomidae (DuretTe-Desset, 1983). Esta especie, localizada en un ejemplar de Artibeus jamaicensis Leach, 1821 proveniente de México, fue descripta originalmente sobre la base de un ejemplar macho por SANDGRound (1929). Posteriormente, CHITwood (1938) describe a la hembra del nematodo aislada de ejemplares de la misma especie de quiróptero.

DURETTE-DESSET \& TCHEPRÁKOFF (1977) examinaron ejemplares de C. globocephala aislados de A. jamaicensis lituratus (actualmente considerada como dos especies válidas: A. jamaicensis y A. lituratus (WILSON \& REEDER, 2005) provenientes de Guyana Francesa. De esta forma complementaron los estudios previos y agregaron nuevos caracteres a la descripción de la misma. Finalmente, Durette-Dessett \& Vaucher (1988) redescriben a la especie y redefinen al género, estudiando ejemplares aislados de A. lituratus y A. planirostris provenientes de Ecuador y Perú. Otros registros centro y sudamericanos de $C$. globocephala corresponden a México, Costa Rica y Nicaragua en A. jamaicensis y Artibeus phaeotis (Miller, 1902) (UBELAKER et al., 1977); México en Artibeus toltecus (Saussure, 1860), Costa Rica y Nicaragua en Carrollia perspicillata (Linnaeus,1758) (UbelaKer et al., 1977); Colombia en A. jamaicensis (Cuartas-Calle \& Muñoz-Arango, 1999), Venezuela en A. jamaicensis, Artibeus cinereus (Gervais, 1856), A. lituratus y C. perspicillata (GuerRero, 1985) y Brasil en A. planirostris (NogueIRA et al., 2004).

Para la Argentina se registró solo al tricostrongílido Allintoshius baudi Vaucher \& Durette-Desset, 1980 parásito de Myotis aelleni Baud, 1979, en la provincia de Chubut (VAuCher \& Durette-Desset, 1980).

En el presente trabajo se describe e ilustra la segunda especie para la ciencia de Cheiropteronema. Asimismo es el primer registro de un nematodo parásito de murciélagos con hábitos frugívoros en la Argentina.

\section{MATERIAL Y MÉTODOS}

Se capturaron 64 ejemplares de A. planirostris (26 hembras, 38 machos) en 16 campañas. Las mismas se realizaron durante febrero, marzo y mayo del 2003; noviembre del 2004; mayo, octubre y noviembre del 2005; enero, julio y noviembre del 2006; febrero del 2007 y mayo del 2008 en localidades de las provincias de Jujuy: El Carmen (24²8'S, 6517’W; 1196 m s.n.m.), Santa Bárbara 


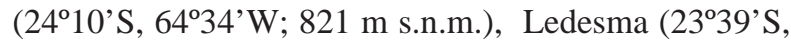
64³2'W; 443 m s.n.m) y Palpalá (24º'15'S, 65²12'W; 1019 m s.n.m.), de Salta: Libertador General San Martín (22 $2^{\circ} 18^{\prime} S$,

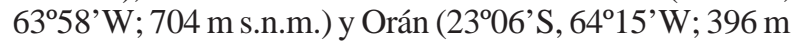
s.n.m) y de Tucumán: Lules (2645'S, 65²8'W; 967 m s.n.m.) y Trancas (26³' $13^{\prime}, 6^{\circ} 17^{\prime} \mathrm{W} ; 1079$ m s.n.m).

Los nematodos, aislados de los murciélagos disecados, fueron fijados con formol al $10 \% \mathrm{y}$ conservados en etanol de $70 \%$. Para el estudio morfométrico fueron diafanizados en lactofenol y dibujados con cámara clara utilizando un microscopio. Las medidas, salvo indicación, corresponden a micras. Se muestra el promedio, desviación estándar y entre paréntesis, valores mínimo y máximo. Los ejemplares observados mediante microscopía electrónica de barrido fueron deshidratados en etanol, tratados con acetonaéter, secados e ionizados con oro. Se calcularon los indicadores de infección parasitaria propuestos por BusH etal. (1997).

Los nematodos identificados fueron incorporados a la Colección Helmintológica de la Fundación Miguel Lillo (CH-FML) y los murciélagos examinados a la Colección Mamíferos Lillo (CML), ambas de Tucumán, Argentina.

\section{Cheiropteronema striatum sp. nov. (Figs 1-20)}

Etimología. El epíteto específico hace referencia a las estrías transversales observadas en la vesícula cefálica.
Diagnosis. Vesícula cefálica estriada (Figs 1, 9, 18), superficie de los huevos con puntuaciones (Fig. 20), espículas iguales, nacimiento simétrico del ala espicular (Fig. 8), número de crestas del synlophe aumenta gradualmente desde la región anterior, alcanza el máximo valor en la región media y disminuye en la posterior (Figs 2-4, 11-13) y no poseen diente esofágico (Figs 10, 17).

Descripción general. Nematodos de gran tamaño, mayores a los dos centímetros y con el cuerpo recto. Poseen una vesícula cefálica más ancha que larga con la superficie estriada transversalmente (Figs 1, 9, 18). Esófago corto, apenas ensanchado en su parte anterior y la posterior más delgada, sin diferenciación de regiones muscular y glandular. El anillo nervioso, poro excretor y deiridios se ubican en la región posterior del esófago (Figs 1,9). En la región cefálica se observa un par de anfidios, cuatro papilas labiales externas y cuatro papilas cefálicas (Figs 10, 17). No poseen diente esofágico.

Synlophe. Las crestas, se disponen regularmente y son similares en tamaño (Fig. 19), se originan a continuación de la vesícula cefálica y se extienden hasta el comienzo de la bursa, en los machos y la cola en las hembras.

En machos y hembras el número de crestas aumenta gradualmente desde la región anterior, alcanza el máximo valor en la región media y disminuye en la posterior.

En machos, en la región anterior, inmediatamente después de la vesícula cefálica, el número de crestas varía entre 26-30, en la del poro excretor: 48, en la región media: 50 y disminuye a 41 el número de ellas en la región del
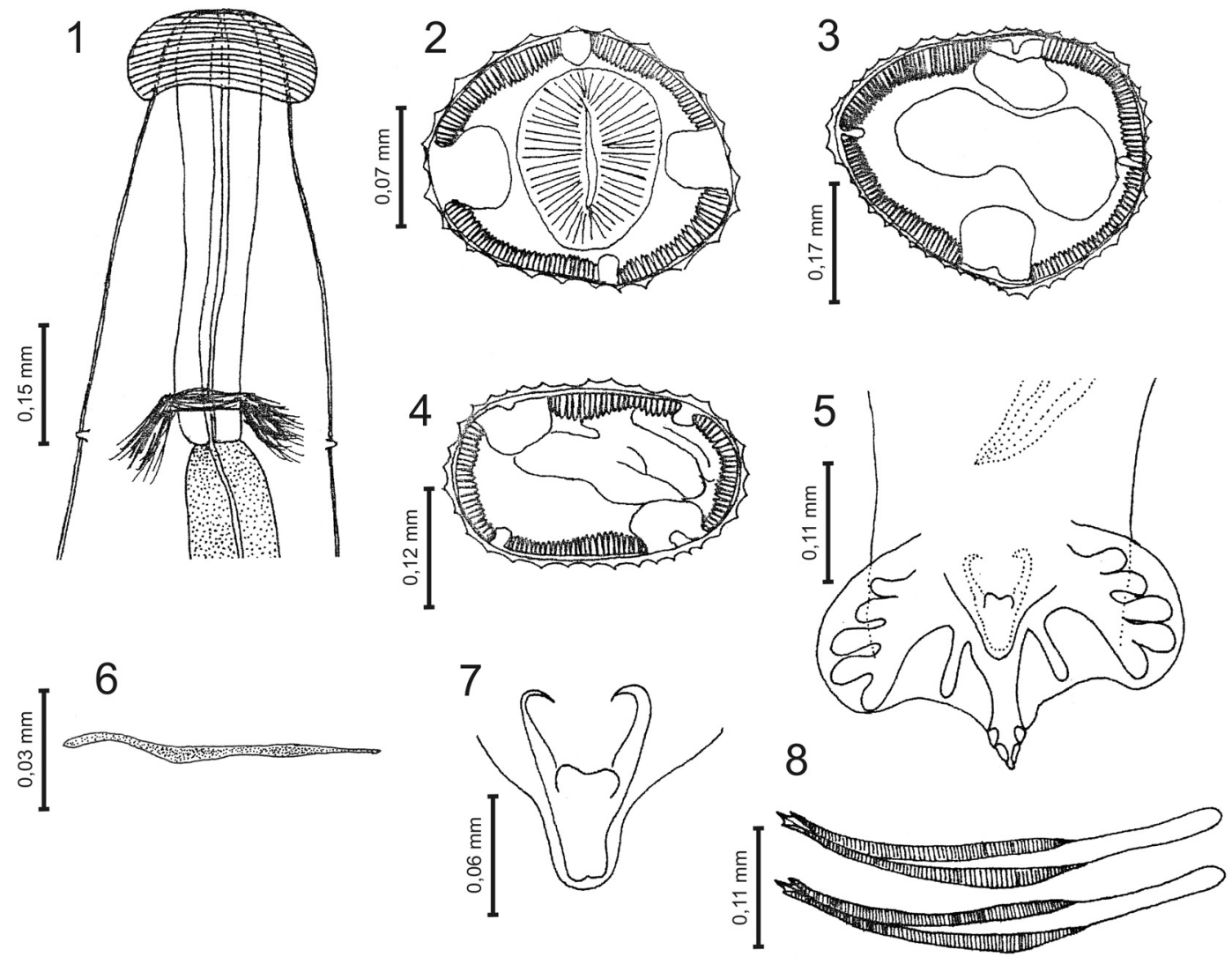

Figuras 1-8. Cheiropteronema striatum sp. nov., macho:1, extremo anterior, vista ventral; 2, corte transversal de la región anterior del cuerpo; 3, corte transversal de la región media del cuerpo; 4, corte transversal de la región posterior del cuerpo; 5, extremo posterior, vista ventral; 6, gobernaculum; 7 , detalle cono genital; 8, espículas. 
inicio de la bursa caudal (Figs 2-4). En hembras, en la región anterior se observan 26 crestas, en la región del poro excretor: 48, en la de la vulva: 58-60 y finalmente en la cola: 36-41 (Figs 11-13).

En los machos la bursa caudal está poco desarrollada en relación a la longitud del cuerpo, posee rayos globosos. Rayo dorsal bien desarrollado, dividido en cinco ramas, dos pares laterales y una rama media alargada de forma triangular; los rayos 8 nacen casi de la base del dorsal. Rayo 2 más corto que el 3 , y éste a su vez más corto que los restantes rayos $(4,5$ y 6 ) (Fig. 5). Cono genital bien desarrollado y complejo, de forma triangular (Fig. 7). El gubernaculum es visible en vista lateral, tiene forma de una lámina delgada, aplanada, ligeramente convexa hacia atrás (Fig. 6). Espículas iguales con la punta trífida, poseen dos alas laterales bien desarrolladas que nacen simétricamente (Fig. 8).

Machos (basado en 10 ejemplares maduros). Largo total $19,40 \pm 4,00(14,54-29,11) \mathrm{mm}$, ancho máximo $500 \pm 20$ (440-510), distancia extremo anterior: anillo nervioso $470 \pm 80(400-680)$, poro excretor $490 \pm 100(400-640)$ y

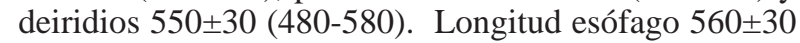
(530-610). Vesícula cefálica: largo 120 \pm 9 (110-140), ancho 230 \pm 20 (200-260). Espículas: largo 410 20 (350-440); cono genital: ancho $90 \pm 7$ (80-100), largo 100 \pm 10 (90-120); bursa caudal: largo 260 \pm 20 (230-290), ancho 360 \pm 70 (250-430); gubernaculum: largo 80 \pm 9 (70-90).

Hembras (basado en 10 ejemplares grávidos). Largo total $23,00 \pm 2,00(20,00-25,5) \mathrm{mm}$, ancho máximo $600 \pm 130$ (440-830), distancia extremo anterior: anillo nervioso $500 \pm 60(330-550)$, poro excretor $500 \pm 50(410-600)$ y deiridios 550 \pm 40 (500-610). Largo esófago 600 \pm 7 (620). Vesícula cefálica: largo 120 \pm 7 (110-130), ancho 240 \pm 30 (200-300). Distancia extremo posterior-vulva 10,50 $\pm 1,85$ $(8,04-12,51) \mathrm{mm}$; longitud: vestíbulo $230 \pm 20(200-270)$,

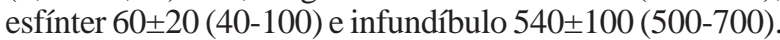
Vagina vera: largo 190 \pm 39 (120-250). Huevo: ancho 60 \pm 6 (50-70), largo $110 \pm 9$ (100-130). Poseen cáscara delgada con puntuaciones en la superficie; en cuyo interior se observan embriones en estadio de dos y cuatro blastómeros (Figs 15, 20). Cola 240 \pm 40 (200-330).

Las hembras son didélficas y el ovijector es anfidélfico; la vulva es postecuatorial, la vagina vera es perpendicular a la pared del cuerpo, con el vestíbulo en forma triangular (Fig. 16). La cola posee cuatro pequeños tubérculos terminales, dos subdorsales, uno ventral y uno medio (Fig. 14).

Material tipo. Holotipo macho CH-FML 07465-A; alotipo hembra CH-FML 07465-B y paratipos (2 machos, 2 hembras) CH-FML 07465-C.

Localidad tipo. Argentina, Jujuy: Santa Bárbara (2410' $\mathrm{S}, 64^{\circ} 34^{\prime} \mathrm{W} ; 821 \mathrm{~m}$ ).

Distribución geográfica. Argentina (Jujuy, Salta y Tucumán).
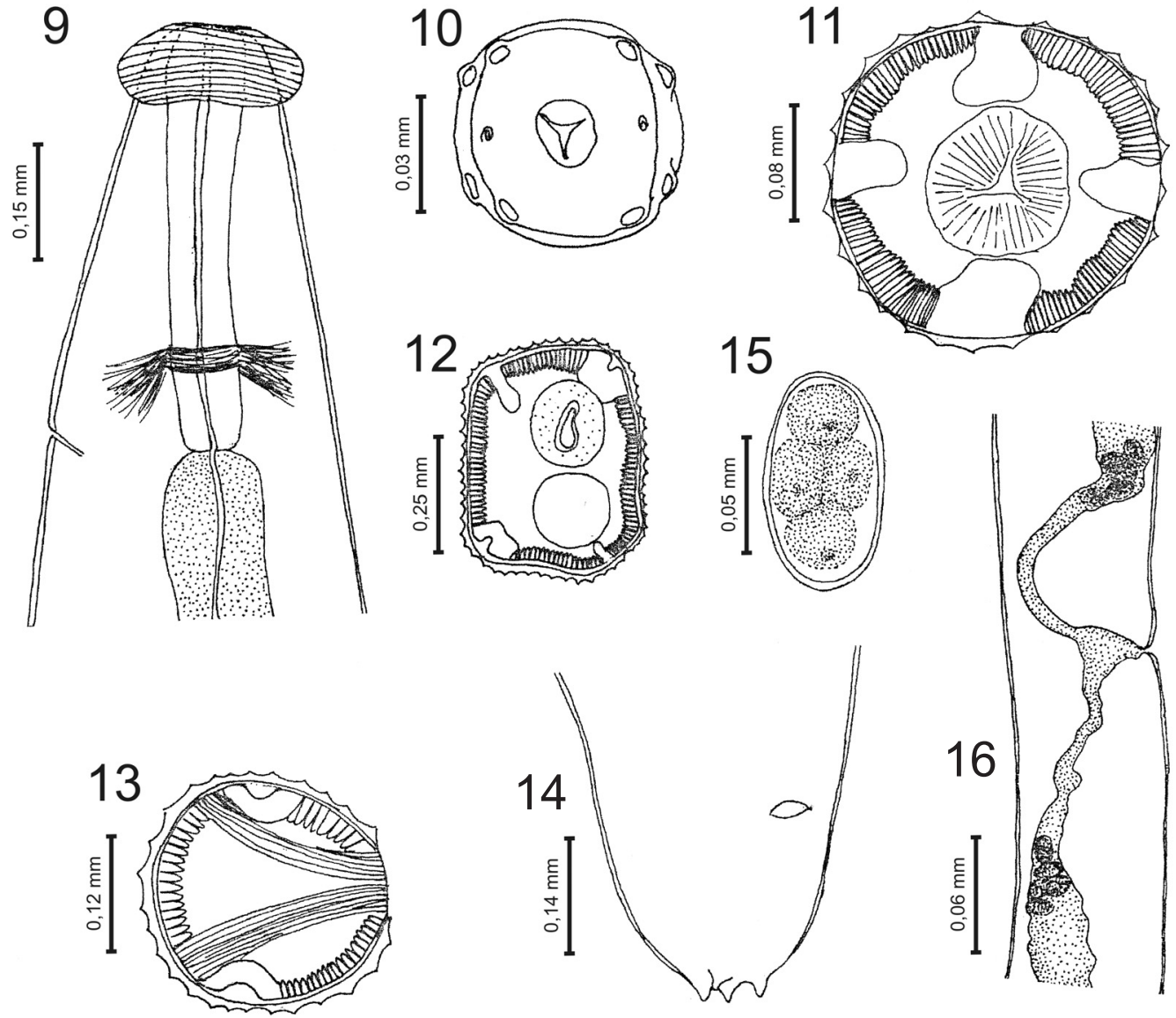

Figuras 9-16. Cheiropteronema striatum sp. nov., hembra: 9, extremo anterior, vista lateral; 10, vista apical; 11, corte transversal de la región anterior del cuerpo; 12, corte transversal de la región media del cuerpo; 13, corte transversal de la región posterior del cuerpo; 14, extremo posterior, vista latero ventral; 15, huevo; 16, región vulvar, vista lateral. 

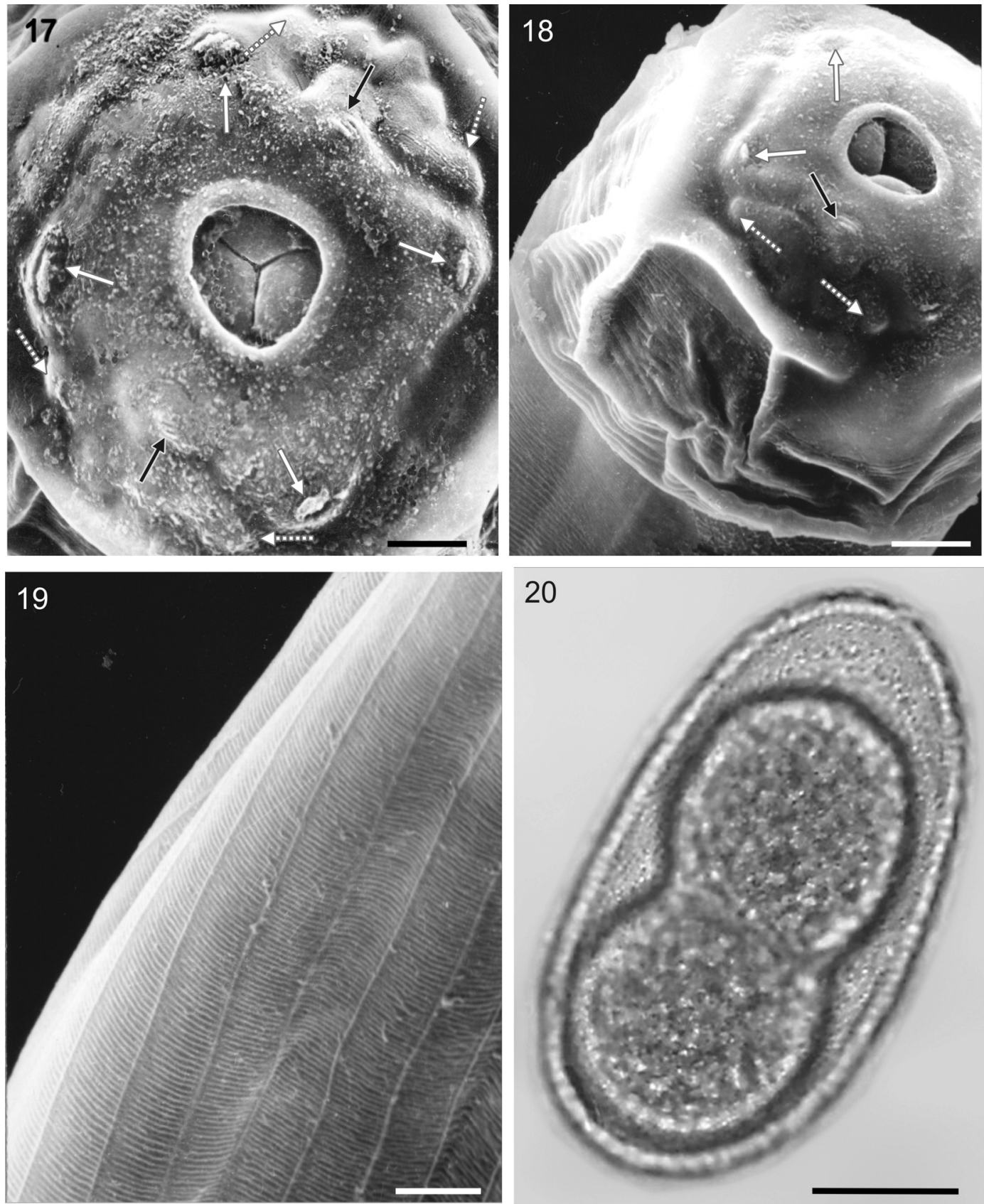

Figuras 17-20. Cheiropteronema striatum sp. nov. 17-19 hembra: 17, extremo anterior, vista apical; 18, vista subapical; 19, estriación cuticular y crestas del synlophe. 20, huevo, medio de montaje: lactofenol. Papilas labiales externas (flechas blancas discontinuas), papilas cefálicas (flechas blancas continuas), deiridios (flechas negras). Escalas: Figs 17-19, $12 \mu$; Fig. $20,24 \mu$.

Hospedador tipo. Artibeus planirostris, CML 07377, capturado en noviembre del 2006.

Localización. Intestino.

Prevalencia. $48 \%$

Intensidad. 71 (43 machos, 28 hembras) de Cheiropteronema striatum sp. nov.

Intensidad media. 2,3 nematodos por murciélago.

\section{DISCUSIÓN}

Los parásitos aquí estudiados presentan los caracteres morfológicos propios del género Cheiropteronema y, además, estructuras que nos permitieron caracterizar una especie nueva. Las diferencias observadas entre Cheiropteronema striatum sp. nov. y $C$. globocephala son: vesícula cefálica (estriada vs. lisa), nacimiento del ala espicular (simétrico vs. asimétrico), número de crestas (aumenta gradualmente desde la región anterior, alcanza el máximo valor en la región media y disminuye en la posterior $v s$. constante a lo largo del cuerpo), espículas (iguales $v s$. subiguales), diente esofágico (ausente vs. presente) y cáscara del huevo (con puntuaciones vs. lisa).

Según la comparación realizada de los caracteres morfométricos de la descripción original y las redescripciones de C. globocephala con las de Cheiropteronema striatum sp. nov., sólo se observan diferencias en la longitud de la vagina vera $(125 \mu$ vs. 30 $\mu)$ y la cola $(100 \mu v s .240 \mu)$ (Tab. I). 
Tabla I. Comparación morfométrica de Cheiropteronema globocephala Sandground, 1929 y Cheiropteronema striatum sp. nov. Las medidas se expresan en micras a excepción de la longitud total en milímetros (DEA, distancia extremo anterior; s/d, sin determinar).

\begin{tabular}{|c|c|c|c|c|c|c|c|c|c|c|}
\hline & \multicolumn{8}{|c|}{ Cheiropteronema globocephala } & \multicolumn{2}{|c|}{$\begin{array}{l}\text { Cheiropteronema } \\
\text { striatum sp. nov. }\end{array}$} \\
\hline & \multicolumn{2}{|c|}{ SANDGROUND, 1929} & \multicolumn{2}{|c|}{ Chitwood, 1938} & \multicolumn{2}{|c|}{$\begin{array}{l}\text { DuRETTE-DESSET \& } \\
\text { TCHÉPRAKOFF, } 1977\end{array}$} & \multicolumn{2}{|c|}{$\begin{array}{c}\text { DURETTE-DESSET \& } \\
\text { VAUCHER, } 1988\end{array}$} & \multirow[b]{2}{*}{$\sigma^{7}$} & \multirow[b]{2}{*}{ q } \\
\hline & $\sigma^{2}$ & q & $\sigma^{2}$ & q & $\sigma^{2}$ & q & $\sigma^{\pi}$ & q & & \\
\hline Longitud total & 19,4 & $\mathrm{~s} / \mathrm{d}$ & 14 & 22,5 & 10,8 & $\mathrm{~s} / \mathrm{d}$ & 20,8 & 26,4 & 19,4 & 23 \\
\hline Ancho máximo & 400 & $\mathrm{~s} / \mathrm{d}$ & 408 & 410 & 330 & $\mathrm{~s} / \mathrm{d}$ & 395 & 500 & 500 & 600 \\
\hline Ancho cápsula cefálica & $\mathrm{s} / \mathrm{d}$ & $\mathrm{s} / \mathrm{d}$ & $\mathrm{s} / \mathrm{d}$ & s/d & 250 & $\mathrm{~s} / \mathrm{d}$ & 215 & 230 & 230 & 240 \\
\hline Largo cápsula cefálica & $\mathrm{s} / \mathrm{d}$ & $\mathrm{s} / \mathrm{d}$ & $\mathrm{s} / \mathrm{d}$ & $\mathrm{s} / \mathrm{d}$ & 150 & $\mathrm{~s} / \mathrm{d}$ & 90 & 125 & 120 & 120 \\
\hline DEA-Anillo nervioso & $\mathrm{s} / \mathrm{d}$ & $\mathrm{s} / \mathrm{d}$ & s/d & 100 & 530 & $\mathrm{~s} / \mathrm{d}$ & 530 & 650 & 500 & 500 \\
\hline DEA- Poro excretor & 480 & $\mathrm{~s} / \mathrm{d}$ & $\mathrm{s} / \mathrm{d}$ & $\mathrm{s} / \mathrm{d}$ & 600 & $\mathrm{~s} / \mathrm{d}$ & 620 & 765 & 500 & 500 \\
\hline DEA- Deiridios & Ausentes & $\mathrm{s} / \mathrm{d}$ & 500 & $\mathrm{~s} / \mathrm{d}$ & 660 & $\mathrm{~s} / \mathrm{d}$ & 650 & 840 & 550 & 550 \\
\hline Largo Esófago & 420 & $\mathrm{~s} / \mathrm{d}$ & s/d & 540 & 580 & $\mathrm{~s} / \mathrm{d}$ & 570 & 630 & 600 & 600 \\
\hline Largo Vagina Vera & & $\mathrm{s} / \mathrm{d}$ & & $\mathrm{s} / \mathrm{d}$ & & $\mathrm{s} / \mathrm{d}$ & & 125 & & 30 \\
\hline Largo Huevos & & $\mathrm{s} / \mathrm{d}$ & & $108-115$ & & $\mathrm{~s} / \mathrm{d}$ & & 110 & & 110 \\
\hline Ancho Huevos & & $\mathrm{s} / \mathrm{d}$ & & $66-75$ & & $\mathrm{~s} / \mathrm{d}$ & & 80 & & 60 \\
\hline Cola & & $\mathrm{s} / \mathrm{d}$ & & 100 & & $\mathrm{~s} / \mathrm{d}$ & & $\mathrm{s} / \mathrm{d}$ & & 240 \\
\hline Largo Espículas & 360 & & 410 & & 440 & & $450-423$ & & 410 & \\
\hline Largo Gobernaculum & 35 & & $\mathrm{~s} / \mathrm{d}$ & & & & 65 & & 100 & \\
\hline
\end{tabular}

Por otra parte C. globocephala posee una amplia distribución, registrándose como parásito de siete especies de murciélagos de los géneros Artibeus y Carollia Gray, 1838 (Phyllostomidae) que habitan naturalmente localidades sudamericanas.

Con respecto a los hospedadores, en Argentina, $A$. planirostris es la única de las tres especies del género de murciélagos registrada en el noroeste del país. Sería conveniente examinar ejemplares de A. planirostris de otras localidades de Sudamérica y de las otras dos especies de Artibeus registradas en el país, para comprobar si Cheiropteronema striatum sp. nov. se distribuye en otras localidades sudamericanas, se restringe al noroeste argentino o bien podría ser registrada en otra región del país y otras especies de quirópteros.

Agradecimientos. A Daniela Miotti por la identificación y provisión de los murciélagos; a Gustavo Sánchez por la digitalización de las figuras. Al Consejo de Investigaciones de la Universidad Nacional de Tucumán (CIUNT) por subsidiar, en parte, la presente investigación. Al personal del laboratorio de Microscopía Electrónica del Noreste Argentino, especialmente a Alberto Andrada Barone por su asistencia técnica.

\section{REFERENCIAS BIBLIOGRÁFICAS}

BARquez, R. M. 2006. Ordem Chiroptera. In: Barquez, R. M.; DíaZ, M. \& Ojeda, R. A. eds. Mamíferos de la Argentina, sistemática y distribución. Tucumán, SAREM. p.56-86.

Barquez, R. M. \& Díaz, M. 2001. Bats of the Argentine Yungas: a systematic and distributional analysis. Acta Zoologica Mexicana, Nueva Serie, 82:29-81.

Barquez, R. M.; Mares, M. A. \& Braun, J. K. 1999. The bats of Argentina. Special Publications, Museum of Texas Tech University 42:1-275.

Barquez, R. M. \& OJedA, R. A. 1992. The bats of the argentine Chaco. Annals Carnegie Museum 61(3):239-261.

Bush, A. O.; Lafferty, K. D.; Lotz, J. M. \& Shostak, A. W. 1997. Parasitology meets ecology on its own terms: Margolis et al. revisited. Journal of Parasitology 83:575-583.

CHitwood, B. G. 1938. Some nematodes from the caves of Yucatán.
Publications of the Carnegie Institute of Washington 491:51-66

Cuartas-Calle, C. \& Muñoz-Arango, J. 1999. Nemátodos en la cavidad abdominal y el tracto digestivo de algunos murciélagos Colombianos. Caldasia 21:10-25.

Durette-Desset, M. C. 1983. Keys to genera of the superfamily Trichostrongyloidea. In: Anderson, R. C. \& Chabaud, A. G. eds. CIH keys to the nematode parasites of vertebrates. Farnham Royal, Commonwealth Agricultural Bureaux. p.1$86, \mathrm{n}^{\circ} 10$.

Durette-Desset, M. C. \& Tchéprakoff, R. 1977. Compléments morphologiques à l'étude de Cheiropteronema globocephala Sandground, 1929. Remarques sur la position systématique et les affinités phylétiques du genre. Bulletin du Muséum National d'Histoire Naturelle 282:1091-1094.

Durette-Desset, M. C. \& Vaucher, C. 1988. Trichostrongyloidea (Nematoda) parasites de Chiroptères néotropicaux. II. Nouvelles données sur le genre Cheiropteronema Sandground, 1929. Revue suisse Zoologie 95:889-899.

Guerrero, R. 1985. Parasitología. In: Aguilera, M. ed. El estudio de los mamíferos en Venezuela: evaluación y perspectivas. Caracas, Fondo Editorial Acta Científica Venezolana. p.35-91.

Hollis, L. 2005. Artibeus planirostris. Mammalian Species 775: $1-6$.

Nogueira, M. R.; Fabio, S. P. \& Peracchi, A. L. 2004. Gastrointestinal helminth parasitism in fruit-eating bats (Chiroptera, Stenodermatinae) from Western Amazonian Brazil. Revista de Biología Tropical 52(2):387-392.

SAndground, J. H. 1929. Some new parasitic Nematodes from Yucatan (Mexico) including a new genus of Strongle from cattle. Bulletin of the Museum Comparative Zoology Harvard 69:515-524.

Simmons, N. B. 2005. Order Chiroptera. In: Wilson, D. E. \& REEDER, D. A. M. eds. Mammal species of the world: a taxonomic and geographic reference. 3ed. Baltimore, Maryland, John Hopkins University. p.312-529.

Ubelaker, J. E.; Specian, R. D. \& Duszynski, D. W. 1977. Endoparasites. In: BAKER, R. J.; JoneS JR., J. K. \& CARTER, D. C. eds. Biology of Bats of the New World Family Phyllostomatidae. Part II. Special publications, 13. Lubbock, Texas Tech University. p.7-56

Vaucher, C. \& DuREtTe-Desset, M. C. 1980. Allintoshius baudi $\mathrm{n}$ sp. (Nematoda: Trichostrongyloidea) parasite du Murin Myotis aelleni Baud, 1979 et redescription de A. tadaridae (Caballero, 1942). Revue suisse Zoologie 86(1):267-278.

Recebido em julho de 2009. Aceito em abril de 2010. ISSN 0073-4721

Artigo disponível em: www.scielo.br/isz 